\title{
A SUPERCONGRUENCE MOTIVATED BY THE LEGENDRE FAMILY OF ELLIPTIC CURVES
}

\author{
HENG HUAT CHAN, LING LONG, AND WADIM ZUDILIN \\ In blessed memory of Anatoly Alekseevich Karatsuba
}

\begin{abstract}
A new supercongruence associated with a Gaussian hypergeometric series, as well as one of Mortenson's supercongruences, are established with new congruence relations and the Legendre transforms of certain sequences.
\end{abstract}

\section{INTRODUCTION}

The Legendre family of elliptic curves

$$
E_{a}: \quad y^{2}=x(x-1)(x-a)
$$

is well known in the literature. For any fixed complex number $a, E_{a}$ corresponds to a double cover of the complex line ramified at four points: $0,1, a$, and $\infty$. If $a$ is different from $0,1, \infty$, the resulting covering curve is an elliptic curve, topologically isomorphic to a torus. Elliptic curve is a well-studied subject and has many important applications such as the Elliptic Curve Cryptography.

Let $p$ be an odd prime and $a$ be $p$-integral not equal to 0 or 1 . Let $\#\left(E_{a} / \mathbf{F}_{p}\right)$ be the number of solutions $E_{a}$ over the finite field $\mathbf{F}_{p}$. From the computations of the Hasse invariant of the elliptic curve $E_{a}$ [7, Chapter V, Section 4], it is known that

$$
\#\left(E_{a} / \mathbf{F}_{p}\right) \equiv-\sum_{k=0}^{(p-1) / 2}\left(\begin{array}{c}
2 k \\
k
\end{array}\right)^{2} 2^{-4 k} a^{k} \quad(\bmod p) .
$$

We mention here that the upper limit of the original sum is $p-1$. However, we may replace $p-1$ by $(p-1) / 2$ since for $k \geq(p+1) / 2$,

$$
\left(\begin{array}{c}
2 k \\
k
\end{array}\right) \equiv 0 \quad(\bmod p) \text {. }
$$

On the other hand, from [2, Sections 2.8-2.11] we know that

$$
\#\left(E_{a} / \mathbf{F}_{p}\right) \equiv-\sum_{k=0}^{(p-1) / 2}\left(\begin{array}{c}
(p-1) / 2 \\
k
\end{array}\right)^{2} a^{k}(\bmod p) .
$$

Combining (1) and (2), we conclude that

$$
\sum_{k=0}^{(p-1) / 2}\left(\begin{array}{c}
2 k \\
k
\end{array}\right)^{2} 2^{-4 k} a^{k} \equiv \sum_{k=0}^{(p-1) / 2}\left(\begin{array}{c}
(p-1) / 2 \\
k
\end{array}\right)^{2} a^{k} \quad(\bmod p) .
$$

The first author was supported by NUS Academic Research Grant R-146-000-103-112. The second author was supported by NSA grant H98230-08-1-0076. 
The congruence (3) can be proved directly by observing that

$$
\left(\begin{array}{c}
(p-1) / 2 \\
k
\end{array}\right)^{2} \equiv\left(\begin{array}{c}
2 k \\
k
\end{array}\right)^{2} 2^{-4 k} \quad(\bmod p) .
$$

Now, when $a=2$, the elliptic curve has the so-called complex multiplication. Roughly speaking, this means $E_{2}$ admits extra symmetries. For instance, if $(x, y)$ is a pair of solution $E_{2}$ then $(-x+2, \sqrt{-1} \cdot y)$ is another. It is known that $E_{2}$ is supersingular over $\mathbf{F}_{p}$ when $p \equiv 3(\bmod 4)$ (see $[7, \S \mathrm{V} .4]$ for definition). Therefore, by the proof of Theorem $4.1[7, \S \mathrm{V} .4]$, if $p \equiv 3(\bmod 4)$ then

$$
\#\left(E_{a} / \mathbf{F}_{p}\right) \equiv 0 \quad(\bmod p) .
$$

Hence, we have the following "modified" version of (3):

$$
\sum_{k=0}^{(p-1) / 2}\left(\begin{array}{c}
2 k \\
k
\end{array}\right)^{2} 2^{-3 k} \equiv(-1)^{(p-1) / 2} \sum_{k=0}^{(p-1) / 2}\left(\begin{array}{c}
(p-1) / 2 \\
k
\end{array}\right)^{2} 2^{k} \quad(\bmod p),
$$

with the factor $(-1)^{(p-1) / 2}$ inserted.

It turns out that the following supercongruence is true:

Theorem 1. Let $p \equiv 1(\bmod 2)$ be a prime, and $n=(p-1) / 2$. Then

$$
\sum_{k=0}^{n}\left(\begin{array}{c}
2 k \\
k
\end{array}\right)^{2} 2^{-3 k} \equiv(-1)^{n} \sum_{k=0}^{n}\left(\begin{array}{l}
n \\
k
\end{array}\right)^{2} 2^{k} \quad\left(\bmod p^{2}\right) .
$$

Our aim of this short note is to prove (7). The surprising fact is that although most proofs of "supercongruences" involve methods like the Wilf-Zeilberger algorithm (see [6], [8]), Gaussian hypergeometric series (such as [1], [6]), hypergeometric series evaluation identities (see, for example, [3]), the proof of (7) requires only some elementary binomial identities.

\section{Proof of Theorem 1}

The proof of Theorem 1 follows from two simple lemmas.

Lemma 1. Let $p$ be a prime and $n=(p-1) / 2$; then

$$
\sum_{k=0}^{n}(-2)^{k}\left(\begin{array}{l}
n \\
k
\end{array}\right)\left(\begin{array}{c}
n+k \\
k
\end{array}\right) \equiv \sum_{k=0}^{n}\left(\begin{array}{c}
2 k \\
k
\end{array}\right)^{2} 2^{-3 k} \quad\left(\bmod p^{2}\right) .
$$

Proof. Note that

$$
\begin{aligned}
\frac{\left(\frac{1}{2}+\varepsilon\right)_{k}}{k !} & =\frac{\left(\frac{1}{2}+\varepsilon\right)\left(\frac{1}{2}+\varepsilon+1\right) \cdots\left(\frac{1}{2}+\varepsilon+k-1\right)}{k !} \\
& =\frac{\left(\frac{1}{2}\right)_{k}}{k !}\left(1+2 \varepsilon \sum_{j=1}^{k} \frac{1}{2 j-1}+O\left(\varepsilon^{2}\right)\right) .
\end{aligned}
$$

Substituting $\varepsilon=p / 2$ and $\varepsilon=-p / 2$ in (9), we find that

$$
\left(\begin{array}{c}
n+k \\
k
\end{array}\right)=\frac{\left(\frac{1}{2}\right)_{k}}{k !}\left(1+p \sum_{j=1}^{k} \frac{1}{2 j-1}+O\left(p^{2}\right)\right)
$$


and

$$
(-1)^{k}\left(\begin{array}{l}
n \\
k
\end{array}\right)=\frac{\left(\frac{1}{2}\right)_{k}}{k !}\left(1-p \sum_{j=1}^{k} \frac{1}{2 j-1}+O\left(p^{2}\right)\right),
$$

respectively. Therefore,

$$
(-1)^{k}\left(\begin{array}{l}
n \\
k
\end{array}\right)\left(\begin{array}{c}
n+k \\
k
\end{array}\right)=\left(\frac{\left(\frac{1}{2}\right)_{k}}{k !}\right)^{2}\left(1+O\left(p^{2}\right)\right) .
$$

After multiplication by $2^{k}$ and summing everything up, we arrive at (8).

The Legendre transform of a sequence $\left\{c_{k}\right\}_{k=0}^{\infty}$ is the sequence $\left\{a_{n}\right\}_{n=0}^{\infty}$ that satisfies the relation

$$
a_{n}=\sum_{k=0}^{n}\left(\begin{array}{c}
n+k \\
k
\end{array}\right)\left(\begin{array}{l}
n \\
k
\end{array}\right) c_{k}, \quad n=0,1,2, \ldots .
$$

To complete the proof of Theorem 1, we will find the Legendre transform of the sequence $(-2)^{k}$.

Lemma 2. The following is true for any non-negative integer $n$ :

$$
\sum_{k=0}^{n}(-2)^{k}\left(\begin{array}{l}
n \\
k
\end{array}\right)\left(\begin{array}{c}
n+k \\
k
\end{array}\right)=(-1)^{n} \sum_{k=0}^{n}\left(\begin{array}{l}
n \\
k
\end{array}\right)^{2} 2^{k}
$$

Proof. Write

$$
\left(\begin{array}{c}
n+k \\
k
\end{array}\right)=\sum_{j=0}^{k}\left(\begin{array}{l}
n \\
j
\end{array}\right)\left(\begin{array}{c}
k \\
k-j
\end{array}\right)
$$

Then

$$
\begin{aligned}
\sum_{k=0}^{n}(-2)^{k}\left(\begin{array}{l}
n \\
k
\end{array}\right)\left(\begin{array}{c}
n+k \\
k
\end{array}\right) & =\sum_{k=0}^{n}(-2)^{k}\left(\begin{array}{l}
n \\
k
\end{array}\right) \sum_{j=0}^{k}\left(\begin{array}{l}
n \\
j
\end{array}\right)\left(\begin{array}{c}
k \\
k-j
\end{array}\right) \\
& =\sum_{j=0}^{n}\left(\begin{array}{l}
n \\
j
\end{array}\right) \sum_{k=j}^{n}(-2)^{k}\left(\begin{array}{l}
n \\
k
\end{array}\right)\left(\begin{array}{c}
k \\
k-j
\end{array}\right) \\
& =\sum_{j=0}^{n}\left(\begin{array}{l}
n \\
j
\end{array}\right)^{2} \sum_{k=j}^{n}(-2)^{k}\left(\begin{array}{l}
n-j \\
k-j
\end{array}\right),
\end{aligned}
$$

since

Therefore,

$$
\left(\begin{array}{l}
n \\
k
\end{array}\right)\left(\begin{array}{c}
k \\
k-j
\end{array}\right)=\left(\begin{array}{l}
n \\
j
\end{array}\right)\left(\begin{array}{l}
n-j \\
k-j
\end{array}\right) .
$$

$$
\begin{aligned}
\sum_{k=0}^{n}(-2)^{k}\left(\begin{array}{l}
n \\
k
\end{array}\right)\left(\begin{array}{c}
n+k \\
k
\end{array}\right) & =\sum_{j=0}^{n}\left(\begin{array}{l}
n \\
j
\end{array}\right)^{2} \sum_{l=0}^{n-j}(-2)^{l+j}\left(\begin{array}{c}
n-j \\
l
\end{array}\right) \\
& =\sum_{j=0}^{n}\left(\begin{array}{l}
n \\
j
\end{array}\right)^{2}(-2)^{j} \sum_{l=0}^{n-j}(-2)^{l}\left(\begin{array}{c}
n-j \\
l
\end{array}\right) \\
& =\sum_{j=0}^{n}\left(\begin{array}{l}
n \\
j
\end{array}\right)^{2}(-2)^{j}(-1)^{n-j}=(-1)^{n} \sum_{j=0}^{n}\left(\begin{array}{l}
n \\
j
\end{array}\right)^{2} 2^{j}
\end{aligned}
$$


The proof of Theorem 1 now follows from (8) and (11).

As a corollary of Theorem 1, we see that the congruence (5) holds.

Remark. In the case $a=1$, the cubic equation $y^{2}=x(x-1)^{2}$ is singular, that is, the curve $E_{1}$ is isomorphic to a sphere but not a torus. This is the degenerate case corresponding to a related supercongruence,

$$
\sum_{k=0}^{(p-1) / 2}\left(\begin{array}{c}
2 k \\
k
\end{array}\right)^{2} 2^{-4 k} \equiv(-1)^{(p-1) / 2} \quad\left(\bmod p^{2}\right),
$$

due to Mortenson [4, Theorem 1] (see also [5, Eq. (1.1)]). It can be also shown using the elementary ideas in the proofs of Lemmas 1 and 2 .

Indeed, summing (10) over $k$ we conclude that

$$
\sum_{k=0}^{(p-1) / 2}\left(\begin{array}{l}
n \\
k
\end{array}\right)\left(\begin{array}{c}
n+k \\
k
\end{array}\right)(-1)^{k} \equiv \sum_{k=0}^{(p-1) / 2}\left(\begin{array}{c}
2 k \\
k
\end{array}\right)^{2} 2^{-4 k} \quad\left(\bmod p^{2}\right)
$$

On the other hand, it is not difficult to see that

$$
\sum_{k=0}^{n}\left(\begin{array}{l}
n \\
k
\end{array}\right)\left(\begin{array}{c}
n+k \\
k
\end{array}\right)(-1)^{k}=(-1)^{n} .
$$

Combining (13) and (14), we arrive at the congruence (12).

It would be nice to have similar elementary proofs for other Mortenson's supercongruences [5, Eqs. (1.2)-(1.4)].

Acknowledgements. The authors would like to thank Robert Osburn, whose conversation with the second author inspired our discovery, and Eric Mortenson for valuable comments. The first and second authors would like to thank National Center for Theoretical Sciences in Hsinchu Taiwan, where the project was started. Also the work was carried out whilst the first author was visiting in the Max Planck Institute for Mathematics (MPIM). He thanks the MPIM for providing a nice research environment.

\section{REFERENCES}

[1] S. Ahlgren and K. Ono, A Gaussian hypergeometric series evaluation and Apéry number congruences, J. Reine Angew. Math. 518 (2000), 187-212.

[2] C. H. Clemens, A scrapbook of complex curve theory, University Series in Math. (Plenum Press, New York, 1980).

[3] D. McCarthy and R. Osburn, A p-adic analogue of a formula of Ramanujan, Arch. Math. (Basel) 91:6 (2008), 492-504.

[4] E. Mortenson, A supercongruence conjecture of Rodriguez-Villegas for a certain truncated hypergeometric function, J. Number Theory 99 (2003), 139-147.

[5] E. Mortenson, Supercongruences between truncated ${ }_{2} F_{1}$ hypergeometric functions and their Gaussian analogs, Trans. Amer. Math. Soc. 355:3 (2003), 987-1007.

[6] R. Osburn and C. Schneider, Gaussian hypergeometric series and supercongruences, Math. Comput. 78 (2009), 275-292.

[7] J. H. Silverman, The arithmetic of elliptic curves (Springer-Verlag, New York, 1986).

[8] W. ZudiLin, Ramanujan-type supercongruences, J. Number Theory 129:8 (2009), 1848-1857. 
Department of Mathematics, National University of Singapore, 2 Science Drive 2, Singapore 117543

E-mail address: matchh@nus.edu.sg

Department of Mathematics, Iowa State University, Ames, IA 50011 USA

E-mail address: linglong@iastate.edu

School of Mathematical and Physical Sciences, University of Newcastle, Callaghan NSW 2308, Australia

E-mail address: wadim.zudilin@newcastle.edu.au 